\title{
Emergency Medical Care and Management of Sports Injuries on the Football Court
}

\author{
Basri Lenjani ${ }^{1 *}$, Premtim Rashiti ${ }^{*}$, Gani Shabani², Arber Demiri ${ }^{1}$, Besarta Pelaj ${ }^{2}$, \\ Arban Demi ${ }^{1}$, Rilind Sylaj ${ }^{1}$, Erza Voca Mulaj ${ }^{3}$, Dardan Lenjani ${ }^{3}$,
}

Received: 26 December 2020 / Accepted: 12 January 2021 / Published online: 20 January 2021

(C) The Author(s) 2021. This article is published with open access at https://journal.astes.org.al

\begin{abstract}
Introduction; Sports medicine is a clinical subspecialty that deals with the examination, monitoring, diagnosis, treatment, and prevention of injuries that occur during sports events, training and physical activities in pre-hospital settings. Managing dramatic situations with minor and multiple injuries is a challenge that requires a quick approach to a dramatic event in managing minor and multiple injuries on the football field and in other sports in support of SHME at pre-hospital and hospital level.

Purpose of the paper. Providing emergency medical care at all basic stages of managing minor and multiple injuries on the football field and in other sports in order to implement BLS, ACLS, BTLS, PTLS, ATLS care measures reducing morbidity, disability, and mortality.

Material and methods. The research is of retrospective, descriptive, qualitative type. The material was taken from the archive of the Emergency Clinic of UCCK for the period January-December 2019. Only the sick or injured in sports matches were taken in the research; Age, gender, type of illness and injury and type of medical care, equipment available, and training and education.

Result. Sports injuries are very costly, and according to the pathology with diseases were 15 cases or $21.4 \%$, injuries were 55 cases or $78 / 6 \%$. Injured by age. The largest number of injured with injuries in the field of football sports the most affected age was the age of $21-25$ years with 28 cases or $40.00 \%$, over 25 years were 27 cases or $38.58 \%$ and with a smaller number were aged $15-20$ years 15 cases or $21.42 \%$.

Discussion and conclusions. A very important factor in sports injuries is the provision of optimal medical care for footballers and other sports in head, neck, spine, chest, abdomen, and pelvis and limb injuries and with a joint communication with the cooperation of health care professionals in the selection of priority cases. Education of medical staff, nurses, paramedics with courses, use of medical equipment, BLS, ACLS, BTLS, PTLS, ATLS as well as standard procedures for providing and transporting medical care to the hospital.

Key word. Illness, injury sports, health care, collision football, medical care, EMS.
\end{abstract}

\section{Introduction}

Key Points: Advancing and empowering EMS through emergency medical care law, sports law and possible national intervention strategy is a very important and priority modality to identify intervention needs for the

Original article, no submission or publication in advance or in parallel

* Corresponding author:

Prof. Ass. Premtim RASHITI, MD. Ph.D.

$\triangle$ premtim.r@gmail.com

1 Emergency Clinic, University Clinical Centre of Kosova, Pristina, KOSOVO

2 College Medical of Sciences Resonance, Pristina, KOSOVO.

3 Faculty of Medicine, University Clinical Centre of Kosovo, Pristina, KOSOVO prevention of sports injuries. Research on the mandatory organization of change, education, training and education to prepare and train health care professionals as well as EMS and after training to be able to provide care and emergency assistance at the right time and moment and this approach can provide new research opportunities aimed at preventing injury and disability and morbidity in sports. Our research was sensitizing to create the awareness of sports clubs should always have a medical team during sports events by invoking the law on medical care, the Law on Sports and the national strategy for injury management in sports. From the research, our evidence has been used extensively in sports injury prevention studies, and to evaluate, modify policy interventions in order to function better.

Sports medicine is a clinical subspecialty undertaken in providing emergency medical care to improve their performance of athletes, in recovering from injuries and to prevent further injuries occurring during sporting events, training and physical activities. Emergency sports medicine 
in mass gatherings provides emergency medical care in minor and mass incidents, sympathizers grouped more than 1000 fans in a football match as well as in other sports. The first priority of any dramatic scene where people's lives are threatened,

EMS are obliged to do the assessment of the scene or the scene to be is safe to enter and can continue assessment, triage and treatment according to priorities.

Multiple disaster management which mainly as its factors of origin of disaster are directly related to the behavior of sympathizers in sports fields. (F-MARC Football Medicine Manual 2nd Edition).

Acute and life-threatening illnesses, injuries and poisonings and require attention to initially stabilize the victims before reaching the SHME. Managing dramatic situations with multiple victims is a challenge that requires ongoing study in theoretical, practical and experiential terms in order to provide clues to an event or disaster. EMS should be present at a dramatic event, but of course unlikely to control the situation but should be prepared and provided in advance, EMS in managing multiple victims in football as well as in other sports when the need arises. (Sports Emergency Care: A Team Approach, Second Edition)

Managing dramatic situations with multiple and minor casualties in sports fields requires the provision of human resources, EMS medical staff, other support services in the management of minor disasters, and sympathizers. (Lenjani Emergency sports medicine 2018).

Factors influencing the management of mass incidents at such sporting events are: Availability, failure to separate sympathizers, failure of match organizers to adhere to guidelines, presence of shutters around the field, EMS access to the field, non-categorization inadequate "risk level" during the match, inadequate access system, insufficient EMS, insufficient barriers, inadequate functioning of the Operations Center, inadequate access system, insufficient trained personnel, inadequate match locations large, the use of tear gas, pre-sale of tickets, matches with large crowds and unreserved seats. (F-MARC Football Medicine Manual 2nd Edition).

The escalation of minor and major risk, based on risk classification is usually based on: mass gathering, the nature of the expected crowd, rivalry of sympathizers, the timing of the football match, the forecast of weather conditions are prerequisites for the occurrence of diseases or injuries can be expected. (F-MARC Football Medicine Manual 2nd Edition).

Moderate risk escalation expected crowds of more than; $50 \%$ of stadium capacity, sports match time day, week, night, meteorological conditions, hot, cold, snow and humidity, nature of sports match, importance, resentment, known rivalry, expected chaos, alcohol present wet conditions / expected rain, sale of regular or black tickets, seats, reserved seats are prerequisites that can generate obvious and very serious problems. (F-MARC Football Medicine Manual 2nd Edition).
Also other factors that may increase the risk are; Insufficient food and drink for spectators, lack of additional capacity, lack of inadequate EMS, lack of emergency exits, lack of adequate signage for basic services in the stadium, provision and intentional use of pyrotechnic devices or tear gas are prerequisites to control the crowd. The number of people seeking integrated EMS during mass gatherings depends on the venue and the legislation of that country and may vary depending on the mass present from 1,000 to 25,000 people or more. (Lenjani Emergency sports medicine 2018).

EMS are obliged within the security perimeter of the stadium and provide medical care including in all areas within the safety zone, scheduled match, lack of previous information, lack of space for action or dysfunctional, lack of adequate communication in the stadium, insufficient education and training of administrators and security staff, insufficient food and drink for spectators.

Mandatory requirements for the football match (obligations from UEFA). The host club is responsible for providing; EMS staff (emergency medicine specialist), at least one stretch for the medical team to arrive at the stadium / hall and until evacuation of the injured player. (Sports Emergency Care: A Team Approach, Second Edition)

EMS components to the mass gathered at the scene requires; adequately educated and trained staff, support staff, physician with appropriate equipment and supported by infrastructure, logistics and mobilization of the integrated system of EMS. (Robb Rehberg Sports Emergency Care (A Team Approach) 3rd Edition)

Medical monitoring and diagnostic equipment which are necessary for the management of diseases and injuries in sports fields which are mandatory and mandatory standards by the home of UEFA and FIFA.

Airway, neck and spinal cord management equipment; manual aspirator, respirators, respirator, lubricant, rigid cervical neck support set. (Lenjani Emergency sports medicine 2018).

Respiratory management equipment; stethoscope, pulse oximeter, oxygen, trauma masks, tubes, pocket masks. mask with bag and valve, separating device for bronchodilators, portable oxygen cylinders. (F-MARC Football Medicine Manual 2nd Edition).

Circulation management equipment; infusion equipment for IV, external automatic defibrillator (AED), equipment for measuring blood pressure with cuffs of suitable sizes, glucometer / test strip. (F-MARC Football Medicine Manual 2nd Edition).

Other (small) equipment; tourniquets, adhesive fixing materials, adhesive fixing materials, light for pupil examinations, IV cannulas of different sizes, solid scissors, disinfectants, disposable gloves, sharp tool boxes, goggles. (F-MARC Football Medicine Manual 2nd Edition).

Emergency medicine bag; adrenaline 1: 10,000 injection, antihistamines, hydrocortisone, benzodiazepines, 
cardiac life-saving drugs, epinephrin or Anapen ${ }^{\circledR}$ (adrenaline autoinjector), bronchodilators, glycerol trinitrate spray, glucose tablets / gel, emergency diabetic drugs and antiemetic (F-MARC Football Medicine Manual 2nd Edition).

Major (mandatory) equipment; spinal trauma carrier, vacuum spoon or mattress stretcher and sufficient fixation equipment, complete set of splints, spinal trauma carrier, vacuum spoon or mattress stretcher and sufficient fixation equipment and complete set of splints. (F-MARC Football Medicine Manual 2nd Edition).

Recommended: The following items should be available; cricothyrotomy set with disposable scalpel equipment for intubation, anti-hypertensive drugs, basket stretcher or stove, external pacing defibrillator and capnography for $\mathrm{CO} 2$ monitoring. (Lenjani Emergency sports medicine 2018).

Emergency medical care Injuries ABC... and per nontrauma CAB. Victim assessment and primary examination 1 minute. CPR and stabilization 5 minutes, Immobilization and transport to hospital 4 minutes. Manage the airways. Control sprains and immobilize head and neck spine and transport. Treatment of sports injuries as follows; Bubbles - impact broken bone break, ice, ligament, elevation. In open and closed fractures and in DRABC ligament injuries and after the stabilization of the vital signs of the injured, emergency transport with medical care to the hospital. In DRABC sprains and after stabilization of vital signs of the injured, emergency transport with medical care to the hospital. (Lenjani First aid-AED 2019). (Lenjani Emergency sports medicine 2018).

In a football stadium is an environment where a large number of people gather, to watch $\bullet$ sports matches, medical emergencies often occur on football fields as well as on sports fields and this component must be managed quickly, effectively and efficiently by EMS. Must be adequate and appropriate medical professionals, cooperative and available to provide necessary medical care. All medical professionals must have education, training and education in a football event (Czarny JM, et al. J Med Ethics 2010; 36: 203-206. Doi: 10.1136 / jme.2009.033621.)

\section{Purpose of the research:}

Providing emergency medical care, prevention, treatment, is necessary to ensure full recovery in the prevention of other injuries in all the basic stages of managing minor and multiple injuries in football and other sports. BLS, ACLS, BTLS, PTLS, ATLS reducing morbidity, disability and mortality. EMS helps prevent, treat, and speed recovery, reduce pain, and promote movement of all injured muscles, restoring normal function, and preventing any recurrent damage or discomfort to the affected tissue. The purpose is also to coordinate medical actions with experienced multidisciplinary medical teams that include surgeons / emergency physicians, orthopedists, cardiologists, nurses.

\section{Material and Methods:}

\subsection{Participants}

The research material was taken from the archive of the Emergency Clinic of UCCK. The research is of retrospective, descriptive, qualitative type, for the period January-December 2019. Only injuries in the field of football sports were taken into research. In the research were taken 70 cases only sick or injured in sports matches; age, gender, type of illness and injury, medical care, available equipment and education. The average age of males was 18-30 years, while according to gender 58 cases for males and 12 cases for females.

\subsection{Variables}

Age and gender were chosen as variables to obtain data on sports injuries. simple demographics for subjects. Types of Injuries was used as a variable to obtain data based on time seasons time series and injuries were categorized into echoes, neck, spinal cord injuries to head and neck, chest, abdomen, upper and lower limbs and pelvis, as a variable for the study were the type of system level injuries.

\subsection{Statistical analysis}

Data were analyzed using the IBM SPSS program. Simple descriptive statistics, tables, diagrams, which were used to explain injury patterns between systems.

\section{Results:}

The research material was taken from the archive of the Emergency Clinic of UCCK only from sports injuries on the football field. The research is of retrospective, descriptive, qualitative type, for the period January-December 2019. (Table 1.)

Table 1. Pathology according to diseases and injuries.

\begin{tabular}{|l|l|}
\hline Pathology & No. (\%) \\
\hline Diseases & $15(21.4)$ \\
\hline Injuries & $55(78.6)$ \\
\hline Total & $70((100)$ \\
\hline
\end{tabular}

Table 2. Number of sick and injured by gender.

\begin{tabular}{|l|l|}
\hline Age & No. $(\%)$ \\
\hline $15-20$ year & $15(21.2)$ \\
\hline $21-25$ years old & $28(40.0)$ \\
\hline Over 26 years old & $27(38.8)$ \\
\hline Total & $70(100)$ \\
\hline
\end{tabular}


Graph 1. Number of sick or injured in sports matches by age.

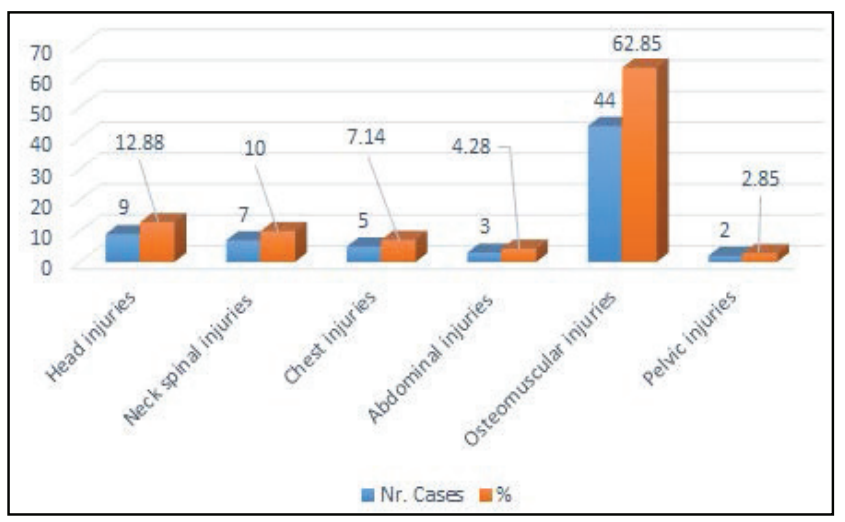

Table 3. Most common diseases.

\begin{tabular}{|l|l|}
\hline Illness & i No. (\%) \\
\hline Convulsive seizures & $4 / 26.6$ \\
\hline Heart attack & $3 / 20.0$ \\
\hline Brain attack & $1 / 6.6$ \\
\hline Hypoglycemia & $4 / 26.6$ \\
\hline Cardiac arrest & $1 / 6.6$ \\
\hline Asthma & $1 / 6.6$ \\
\hline Total & $15 /$ \\
\hline
\end{tabular}

Table 4. Number of educated and trained medical staff managing sports clubs.

\begin{tabular}{|l|l|}
\hline Compulsory courses & No. (\%) \\
\hline BLS & 24 \\
\hline ACLS & 4 \\
\hline BTLS & $/$ \\
\hline ATL & $/$ \\
\hline PHTLS & $/$ \\
\hline Total & 28 \\
\hline
\end{tabular}

Table 5. Equipment ambulance EMS.

\begin{tabular}{|l|l|}
\hline Equipment Ambulance EMS & \\
\hline Ambulance BLS & $75 / 78.9$ \\
\hline Ambulance ACLS & $5 / 5.2$ \\
\hline Ambulance no-equipment & $15 / 15.9$ \\
\hline Total & $95 / 100$ \\
\hline
\end{tabular}

Table 6. EMS Transport private and EMS.

\begin{tabular}{|l|l|}
\hline EMS Transport & No. (\%) \\
\hline Privat & $15(21.4)$ \\
\hline EMS & $55(78.6)$ \\
\hline Total & $70 / 100$ \\
\hline
\end{tabular}

\section{Discussion and Conclusion:}

The results of our study showed that problems appear in different steps during the management of diseases and injuries in sports fields in the absence of mobile teams, capacities, human resources, medical equipment, triage areas and their system. Lack of coordination of actions was the main cause, delays in the management of the sick and injured on sports fields.

Injured by age the largest number of injured with injuries in the field of football sports the most affected age was the age of $21-25$ years with 28 cases or $40.00 \%$, over 25 years were 27 cases or $38.58 \%$ and with a smaller number were aged $15-20$ years 15 cases or $21.42 \%$.

Injured according to pathology with osteo-muscular injuries were 44 cases or $62.8 \%$, with head injuries were 9 cases or $12.8 \%$, with neck and spinal injuries were 7 cases or $10.0 \%$, with chest injuries were 5 cases $7.1 \%$, with abdominal injuries were 3 cases or $4.2 \%$ and with pelvic injuries were 2 cases or $2.8 \%$.

The most common diseases according to frequency with Convulsive seizures 4 cases or $26.6 \%$ with heart attack 3 cases or $20.0 \%$, brain attack1 cases or $6.6 \%$, hypoglycemia 4 cases or $26.6 \%$, cardiac arrest 1 case or $6.6 \%$ and with asthma 1 case or $6.6 \%$. These cases were among the sympathizers. All these cases were hospitalized after admission, monitoring, diagnostic observation and treatment were placed in the appropriate clinics according to the nature of the disease.

All cases were transported by private cars without care and medical transport, because EMS were insufficient. EMS ambulances with BLS medical equipment were 75 ambulances or $78.9 \%$ with ACLS 5 ambulances or $5.2 \%$ and EMS ambulances not equipped with medical equipment were 15 ambulances or $15.9 \%$. It is required that EMS should be modernized respecting the standards of medical care transport based on the law of medical care as in education, education and training with basic courses for outpatient service, but this component is not developed, so we must review the current laws that regulate EMS. Medical treatment at the scene was low-key because the medical teams did not have any medical training and most of them were uneducated, educated and trained with compulsory courses and the triage system was deficient in assessing the condition of the patients. and the injured.

As for the national medical team, they have been trained by the UEFA Medical Committee, but among them are not professional medical teams and in their composition is a pediatric surgeon and two physiotherapists, but bypassed the emergency doctor, orthopedic, anesthesiologist, cardiologist, nurses who have been eliminated, not respecting the recommendations of UEFA and FIFA. This issue should be regulated by the National Football Federation of Kosovo.

A very important factor in sports injuries is the provision of optimal medical care for footballers and in other sports in head, neck, spine, chest, abdomen, pelvis and limb injuries as well as joint communication in collaboration 
with health care professionals in selection of priority cases. (F-MARC Football Medicine Manual 2nd Edition). National Sports Federations of Kosovo based on the rules written by UEFA, FIFA national sports teams must manage doctors' professional multidisciplinary medical teams such as emergency physicians, anesthesiologists, sports doctors, traumatologists, cardiologists, nurses, educated physiotherapists, trained and certified with the required courses BLS-D, PHTLS, ACLS, ATLS, T-triage.

Education of medical staff, nurses, paramedics with courses, use of medical equipment, BLS, ACLS, BTLS, PTLS, ATLS as well as standard procedures for providing and transporting medical care to the hospital. The Sports Law of the Republic of Kosovo, No. 2003/24 - XIV, must be supplemented and amended to meet the recommendations of FIFA and UEFA for emergency medical care in sports, including Article 60 of this Law on Health Protection and the use of stimulant substances and methods in sports.

The results of this research work suggest that the National Federation of Kosovo should oblige sports clubs to provide EMS during training and during the organization of sports games, to prevent treatment and wanting to achieve an optimal recovery. The establishment of the national center and the national strategy is immediate is a valuable reflection of the scope of our research in the prevention of sports injuries.

COI Statement: This paper has not been submitted in parallel. It has not been presented fully or partially at a meeting or podium or congress. It has not been published nor submitted for consideration beforehand.

This research received no specific grant from any funding agency in the public, commercial, or nonprofit sectors. There are no relevant or minor financial relationships from authors, their relatives or next of kin with external companies.

Disclosure: The authors declared no conflict of interest. No funding was received for this study.

Acknowledgement: We would like to thank medical staff of Emergency Clinic of the University Clinical Center of Kosovo.

\section{References}

1. Buss DD, Lynch GP, Meyer CP, Huber SM, Freehill MQ. Nonoperative management for in-season athletes with anterior shoulder instability. Am J Sports Med. 2004; 33:1430-4.

2. Gardiner JR, Madaleno JA, Johnson DL. Sideline management of acute knee

3. injuries. Orthopedics. 2004; 27:1250-1254.

4. Mangine RE, Minning SJ, Eifert-Mangine M, Colosimo AJ, Donlin M. Management of the patient with an ACL/MCL injured knee. N Am J Sports Phys Ther. 2008; 3: 204-11.

5. Emergency medicine in football is now regarded as a subspeciality of emergency medicine. EFRAIM B KRAMER, MB BCh, BSc (Hons), FCEM (SA), Dip PEC(SA), CME May 2010 Vol.28 No.5. P.205-110. 2010.

6. Lenjani.B Basic Emergency Support of Life - AED, P.15-4060, 199-210,2017

7. Recognition and Management of Sporting Emergencies: An Introduction. PY-4019 Impairment and Disability Sports Group Andrew Storan Darren Hickey David Niblock Maria McMahon Noel O Reilly Siobhan Culle. P.205-1102, 5-2-3745=60, 2013

8. Petersen W, et al. Treatment of acute ankle ligament injuries: a systematic review. Arch Orthop Trauma Surg. 2013; 133: 1129-1141.

9. Krutsch, W., Mayr, H.O., Musahl, V., Della Villa, F., Tscholl, P.M., Jones, H. Injury and Health Risk Management in Sports, 2nd Edition P. 34-45.

10. Lenjani. B Urgent Medical Assistance and medical condition special - Emergency Urgency. P.9-25-40.90- 190-220, 2018

11. Lenjani. B First aid-AED, P.20-20-50.80-110. 2019.

12. National championships medical minimum standards P. $3=15$. 2019-202.

13. Schwellnus M, IOC Medical Commission, International Federation of Sports Medicine. The Olympic textbook of medicine in sport. Oxford, UK; Hoboken, NJ: WileyBlackwell; 2008.Mo Farah speaks to the BBC following his 5000m win 2012 London Olympics. From November 2013.

14. Elizabeth Quinn, Medically, Michael Menna, Health and safety Sports Injury First Aid Treatment September P. 3-735-7017, 2020 